\begin{tabular}{|c|l|}
\hline Title & Phason-flips refinement of and multiple scattering correction for the d-A ICuRh quasicrystal \\
\hline Author(s) & Ruganski, Ireneusz; Strzal ka, Radoslaw; Wolny, Janusz \\
\hline Citation & $\begin{array}{l}\text { Acta crystallographica. Section A, Foundations and advances, 75, 352-361 } \\
\text { https://doi.org/10.1107/S2053273318017254 }\end{array}$ \\
\hline Issue Date & 2019-03 \\
\hline Doc URL & http://hdl.handle.net/2115/73541 \\
\hline Rights & ○ International Union of Crystallography \\
\hline Type & article \\
\hline File Information & buganski_reprint.pdf \\
\hline
\end{tabular}

Instructions for use 


\section{Phason-flips refinement of and multiple-scattering correction for the $d$-AICuRh quasicrystal}

\section{Ireneusz Buganski, Radoslaw Strzalka and Janusz Wolny}

Acta Cryst. (2019). A75, 352-361

\section{IUCr Journals CRYSTALLOGRAPHY JOURNALS ONLINE}

Copyright (C) International Union of Crystallography

Author(s) of this article may load this reprint on their own web site or institutional repository provided that this cover page is retained. Republication of this article or its storage in electronic databases other than as specified above is not permitted without prior permission in writing from the IUCr.

For further information see http://journals.iucr.org/services/authorrights.html 


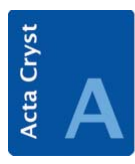

FOUNDATIONS

ADVANCES

ISSN 2053-2733

Received 29 May 2018

Accepted 4 December 2018

Edited by A. Altomare, Institute of Crystallography - CNR, Bari, Italy

Keywords: phason flips; multiple scattering; quasicrystals; structure refinement.

Supporting information: this article has supporting information at journals.iucr.org/a

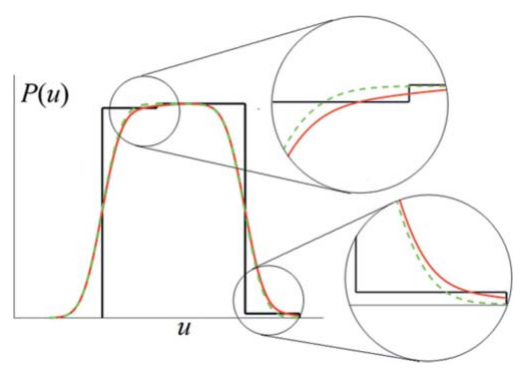

(C) 2019 International Union of Crystallography

\section{Phason-flips refinement of and multiple-scattering correction for the $d$-AICuRh quasicrystal}

\author{
Ireneusz Buganski, ${ }^{\mathrm{a}, \mathrm{b} *}$ Radoslaw Strzalka ${ }^{\mathrm{a}}$ and Janusz Wolny ${ }^{\mathrm{a}}$ \\ ${ }^{\mathrm{a}}$ Faculty of Physics and Applied Computer Science, AGH University of Science and Technology, Krakow, Poland, and \\ ${ }^{\mathbf{b}}$ Graduate School of Engineering, Division of Applied Physics, Hokkaido University, Sapporo, Japan. ${ }^{*}$ Correspondence \\ e-mail: ireneusz.buganski@fis.agh.edu.pl
}

The origin of the characteristic bias observed in a logarithmic plot of the calculated and measured intensities of diffraction peaks for quasicrystals has not yet been established. Structure refinement requires the inclusion of weak reflections; however, no structural model can properly describe their intensities. For this reason, detailed information about the atomic structure is not available. In this article, a possible cause for the characteristic bias, namely the lattice phason flip, is investigated. The derivation of the structure factor for a tiling with inherent phason flips is given and is tested for the $\mathrm{AlCuRh}$ decagonal quasicrystal. Although an improvement of the model is reported, the bias remains. A simple correction term involving a redistribution of the intensities of the peaks was tested, and successfully removed the bias from the diffraction data. This new correction is purely empirical and only mimics the effect of multiple scattering. A comprehensive study of multiple scattering requires detailed knowledge of the diffraction experiment geometry.

\section{Introduction}

Quasicrystals have been known for more than 30 years (Shechtman et al., 1984) and still fascinate researchers with their complex structures and unusual geometries. Many questions remain unanswered, even for a standard problem such as structure determination. Although the methods of structure refinement employed in crystallography have become mature and almost fully automated throughout decades of studies (see e.g. Panjikar et al., 2009; Cascarano et al., 1991; Skubák \& Pannu, 2013), this is not the case for the structure refinement of quasicrystals. The process of determining a structure model is non-trivial and requires the researcher's attention at every step of the structure solution, especially in the initial stages (see e.g. Takakura et al., 2007; Ors et al., 2014). Although around 50 stable quasicrystalline systems are known, only a few structures have been solved quantitatively (Steurer, 2018). Much has been done for decagonal quasicrystals, which are majorly represented among the structures with a 'forbidden' symmetry (Steurer \& Deloudi, 2009). In earlier work, a structure model based on Penrose tiling used as a quasilattice and providing long-range order enabled us to achieve an agreement within six orders of magnitude in the intensities of the calculated/measured diffraction data (Kuczera et al., 2012, 2014). However, for small peaks, the deviation from the measured intensities is still much larger than the error of measurement. This signifies that there is a structural effect or defect not included in the refinement procedure. The broadening observed at the level of intensities below $10^{-4}$ relative to the strongest reflection in the 
correlation plot of experimentally observed structure factors suggests that current models must be revised to include more sophisticated types of disorder. Also in this range, a characteristic bias of the structure factor arises, becoming more prominent for weaker diffraction reflections. In previous articles, we suggested that a possible reason for such a deviation might be the general Debye-Waller factor in the standard, exponential form (Lubensky et al., 1986; Bancel, 1989) used to correct the model for the effect of phason fluctuations (Buganski et al., 2015). The general Debye-Waller factor is theoretically justified only for the random-tiling types of structures (Henley et al., 2000; Buganski et al., 2017). Despite this, it is broadly applied to the structure refinement of all kinds of quasicrystals and is essential for a successful convergence of the calculation. Another potential reason for the characteristic bias observed in the correlation plot might be multiple scattering, which increases the intensities of weaker reflections (Takakura \& Mizuno, 2015; Fan et al., 2011). Multiple scattering is particularly difficult to handle as the eventual correction is supposed to be applied at the dataacquisition stage. In this article, we propose an improvement of the model for the AlCuRh decagonal quasicrystal that allows for the integration of lattice phason flips during the calculation of the structure factor. The new correction for phasons is shown to work as necessary alongside the general Debye-Waller factor. Additionally, empirical corrections will be used to remove the effect of the intensity bias (caused by multiple scattering) by redistributing the intensities of the peaks.

\section{The Penrose tiling}

The Penrose tiling was invented by Roger Penrose in 1974 as an answer to the problem of the aperiodic coverage of a 2D plane (Penrose, 1974). The problem was phrased: does there exist a finite set of tiles that can cover a 2D plane without gaps or overlaps and which does not form a periodic pattern? It was shown that the problem can be solved by the use of two rhombuses. Penrose tiling appeared to be indispensable for the investigation of quasicrystalline structures, as it provides an example of long-range order without translational symmetry. The point symmetry of the Penrose tiling diffraction pattern has the classically forbidden tenfold rotational symmetry, also observable in quasicrystals. The atomic structure built upon the tiling fits the experimental X-ray diffraction data and electron microscope images perfectly. This was partly a proof of the existence of the quasiperiodic atomic systems in nature (e.g. Abe \& Tsai, 2001; Taniguchi \& Abe, 2008).

The mathematical construction of the Penrose tiling can be achieved by three methods: using the de Bruijn construction (de Bruijn, 1981), applying inflation rules (Henley, 1986) and by a projection from a higher-dimensional space, which can be used to obtain a generalized class of tilings (Pavlovitch \& Kleman, 1987). The last is of great importance, as the prevalent method of the structure description of quasicrystals involves exploiting a higher-dimensional space and provides an analytical method of calculating the structure factor of the decagonal quasicrystal modelled with the Penrose tiling as a quasilattice. The higher-dimensional model of the Penrose tiling can be obtained using the 4D and 5D embedding (Steurer \& Deloudi, 2009). We chose the 5D embedding for a clear distinction between the distribution of the rhombuses expressed by the $z_{\perp}$ coordinate in the perpendicular space. We discuss this subject more extensively later in this article. A 5D space is spanned by $\mathbf{d}_{i}$ vectors, $i=\{1,2, \ldots, 5\}$ :

$$
\begin{aligned}
\mathbf{d}_{i}= & a\left(\cos \left[\frac{2 \pi}{5}(i-1)\right] \quad \sin \left[\frac{2 \pi}{5}(i-1)\right] \quad \cos \left[\frac{4 \pi}{5}(i-1)\right]\right. \\
& \left.\sin \left[\frac{4 \pi}{5(i-1)}\right] \quad \frac{1}{2^{1 / 2}}\right),
\end{aligned}
$$

forming a 5D cube. The parameter $a$ is the edge length of the rhombus in a $2 \mathrm{D}$ plane. The first two coordinates define the parallel space and the last three define the perpendicular space. The spaces are mutually orthogonal. The real-space positions of the Penrose tiling are obtained by projecting all points of a 5D space, generated by integer addition of the $\mathbf{d}_{i}$ vectors, onto the parallel space. Only points that fit the projection window (also called the atomic surface or the occupation domain) are taken. The projection window is an object in the perpendicular space encompassing one unit cell of 5D space [for details of the mathematics of the projection method, see e.g. Duneau \& Katz (1985)]. In the case of the Penrose tiling in the 5D embedding, the projection window has the shape of four regular pentagons lying one over another along the $z_{\perp}$ direction a distance of $a / 2^{1 / 2}$ apart. All the nodes of the Penrose tiling (vertices of rhombuses) lie inside the projection window. The advantage of such an approach is that an infinite and discrete set of points in real space is represented in the perpendicular space by compact objects that are easy to integrate at the structure-factor calculation stage. The Penrose tiling in real space can be represented by two rhombuses: 'thick', labelled $L$, and 'thin', labelled $S$. We introduce a special labelling of rhombuses with a different orientation. Since each rhombus is spanned by two of the five vectors $\mathbf{d}_{i}$ (Fig. 1) radiating from one vertex (a reference vertex), e.g. vectors $\mathbf{d}_{3}$ and $\mathbf{d}_{4}$, we can use the subscript ' 34 ' for the specific thick rhombus $L_{34}$. The thin rhombus spanned by vectors $\mathbf{d}_{2}$ and $\mathbf{d}_{5}$ will be called $S_{25}$. We do not distinguish an interchange in the subscript ordering (e.g. $S_{25}$ and $S_{52}$ denote the same object).

Figure 1

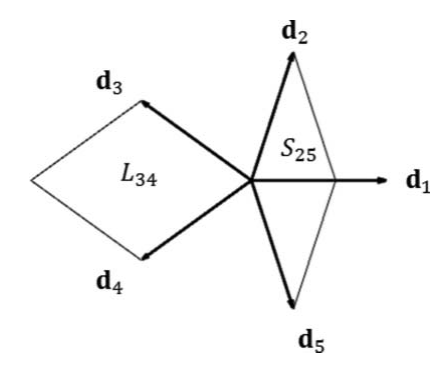

Definition and labelling of the rhombuses in the Penrose tiling by 5D basis vectors. 


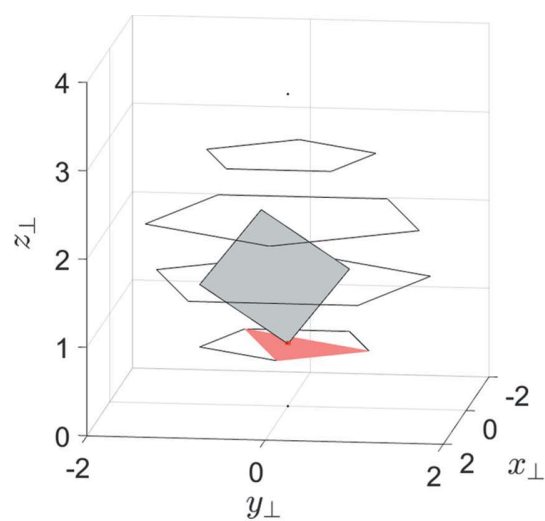

(a)

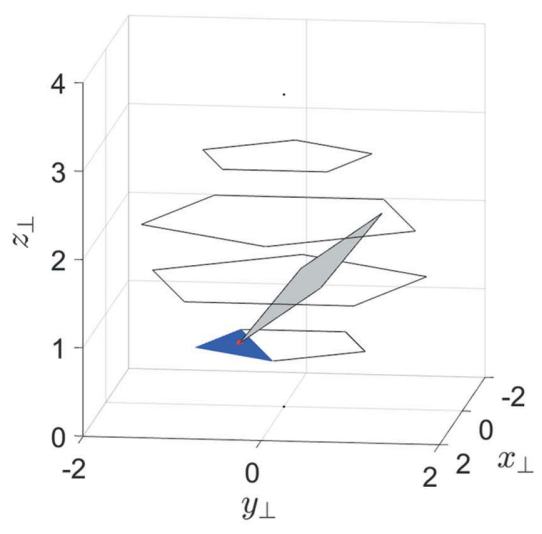

(b)

Figure 2

The distribution of the reference vertex of $(a)$ the thick rhombus and $(b)$ the thin rhombus formed on the first pentagon of the atomic surface. An analogous distribution is formed on the second pentagon. The coordinates $x_{\perp}, y_{\perp}$ and $z_{\perp}$ are associated with the third, fourth and fifth coordinates of the vectors $\mathbf{d}$, respectively.

All rhombuses may occur in five different orientations, but in the perpendicular space an additional distinction can be achieved. The reference vertices of all rhombuses in a given orientation have a perpendicular-space representation that forms two triangular distributions lying on the first and second pentagon of the atomic surface (Fig. 2). Because the distribution in perpendicular space is formed by two nonoverlapping triangles, two classes of rhombuses can be distinguished depending on in which distribution the reference vertex has its representation. This distinction is crucial to properly calculate the structure factor. If distributions of the reference vertices are found for each type of rhombus, the structure factor $F(\mathbf{k})$ can be calculated as follows (Kozakowski \& Wolny, 2010):

$$
\begin{aligned}
F(\mathbf{k}) & =D_{\text {phas }}\left(\mathbf{k}_{\text {perp }}\right) \\
& \times \sum_{c} \sum_{t} \sum_{o=1}^{5}\left[F_{t}^{o, c}(\mathbf{k}) \sum_{j=1}^{N_{t, c}} p_{j}^{t, c} f_{j}^{t, c} D_{j}^{t, c}(\mathbf{k}) \exp \left(i \mathbf{k r} \mathbf{r}_{j}^{t, c, o}\right)\right],
\end{aligned}
$$

where $\mathbf{k}$ is the parallel component of the scattering vector, $F_{t}^{o, c}(\mathbf{k})$ is the Fourier transform of the triangular subregion corresponding to the rhomb ( $t=$ thick, thin) in the given orientation $o$ and class $c$ (the first or second pentagon), $\mathbf{r}_{j}^{t, c, o}$ is the position of the atom $j$ in the rhomb $t$ for the orientation $o$ and class $c, p_{j}^{t, c}$ is the fraction of the atom $j$ inside the rhomb $t$, $N_{t, c}$ is the number of atoms decorating the rhomb $t$ of class $c$ and $f_{j}^{t, c}$ is the atomic form factor. Two corrections are made for the atomic disorder. $D_{j}^{t, c}(\mathbf{k})$ corrects for atomic anisotropic displacement parameters (ADPs) and a phononic Gaussian Debye-Waller formula is used as the correction term. The second factor is defined by the general Debye-Waller factor $D_{\text {phas }}\left(\mathbf{k}_{\text {perp }}\right)$ calculated for the whole structure and is responsible for the correction for the phasons. Equation (2) is currently widely used for the refinement of decagonal quasicrystals modelled with the Penrose tiling as a quasilattice. For the purposes of our study, we must modify the lattice component $F_{t}^{o, c}(\mathbf{k})$ in equation (2), which is affected by the phason flips. Apart from the use of non-overlapping Penrose rhombuses, it is also possible to cover a $2 \mathrm{D}$ space with clusters of a specific shape (Duda et al., 2007), resulting in the same point symmetry. The derivation of the structure factor and a subdivision of the statistical domain in the average unit cell (AUC) (Wolny, 1998; Wolny et al., 2017) for a cluster-based decagonal structure has been carried out by Dąbrowska et al. (2005).

The periodicity of the quasicrystal can be restored by the higher-dimensional approach, as discussed above for the Penrose tiling, or by the use of the AUC approach. In the latter approach, the real-space coordinates of atoms or quasilattice nodes are projected onto a periodic reference lattice, giving a distribution of atomic (node) positions. It can be shown that the coordinates in the statistical approach are related by strict rules to the perpendicularspace coordinates, providing a clear interpretation of the atomic surface (Wolny et al., 2002). This is true as long as the phonons are not part of the model and are excluded from the distribution (Wolny et al., 2016). In this article, we limit the discussion to phason effects, therefore the notion of the distribution in the AUC is fully equivalent to the notion of the atomic surface in the higher-dimensional approach. Note that the atomic distribution was previously used to explain the compactness of the regions of reference vertices within the atomic surface, although the notion of the atomic distribution is not a part of the higher-dimensional nomenclature explicitly. The two notions of the AUC and the atomic surface are, however, equivalent in our case. The representation of the atomic surface is more common in the literature, and for this reason we selected it to explain the atomic-surface subdivision mechanism. The second reason is that the coordinates in the AUC can be calculated directly from the coordinates in the perpendicular space for any chosen reference lattice, which helps to avoid confusion.

\section{Phason flip}

The phason flip is an intrinsic part of the quasiperiodic structure, restricted by the geometry of a quasicrystal to the specific rearrangement of atoms or structural units (Janssen \& Radulescu, 2003; Kiselev et al., 2012). Note that a few different defects are called phasons in the literature. We limit our use of the term to the case of the phason flips only. The phason flip interchanges the positions of building blocks of the quasilattice in a specific manner characteristic for the individual type of quasicrystal. The rule is that the outer boundaries of the flippable pattern must be preserved (Figs. 3 and 4). The simplest flippable pattern in the Penrose tiling is a combination of two thick rhombuses and one thin rhombus, or two thin rhombuses and one thick rhombus. When the flip is introduced, the resulting pattern is the inversion of the initial one with respect to the centre of the parallelogram constructed from the outer sides of the rhombuses (which is a hexagon in 
this case). The flip is graphically explained in Figs. 3 and 4. The shift of the node $O$ to position $O^{\prime}$ is the simplest implementation of the phason flip. As a result, the configuration of rhombuses inside the hexagon is changed to form an inverted pattern. The flip does not cause any overlap between neighbouring tiles or voids. The shape and the orientation of the parallelogram are preserved, only the internal pattern is altered. However, the phason flip introduces a change in the AUC distribution, depending on the type of flip. Part of the distribution is relocated to a new position by the flip vector, which is defined as the translation vector of the inner node in the flippable pattern (the vector from the point $O$ to the point $\left.O^{\prime}\right)$. The part of the distribution that is related to the inner node position is shifted. The height of the cut in the distribution is proportional to the flip probability. For the nondecorated Penrose tiling, in which point-scattering sources with a uniform, k-vector-independent scattering power are located at the vertices of the Penrose tiling, there are two types of relocation and, as a consequence, two basic kinds of change in the AUC distribution can be considered. The cut appears in the distribution belonging to the first or second pentagon, depending on the type of flip. In Fig. 3, we visualize the phason flip in the pack of two fat rhombuses and one thin rhombus (fft - fat, fat, thin) and its impact on the AUC distributions. The red prism represents the region corresponding to the distribution of the reference vertex $O$. The height of the prism is proportional to the flip probability
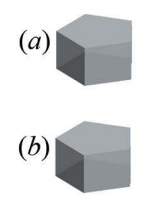

(c)
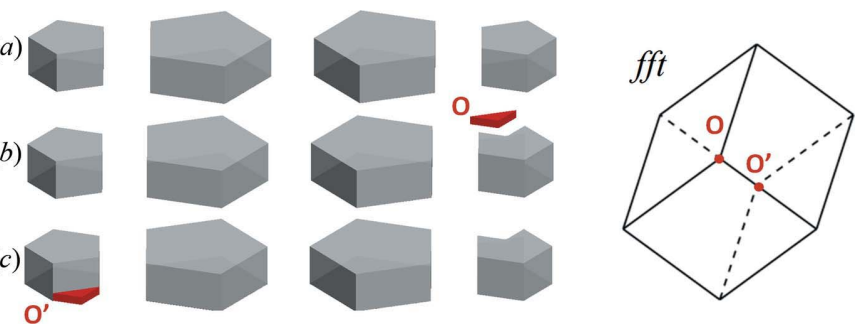

Figure 3

The redistribution of the AUC caused by the phason flip in one orientation of the patch $f f t$. The original configuration (with the inner node $O$ ) is flipped by shifting the node $O$ to the position $O^{\prime}$. This results in a shift of the region in the AUC representing points $O$ (forming a prism) to a new, previously unoccupied region $O^{\prime}$. The height of the prism is proportional to the flip probability, which is equal to 0.2 in this figure. A scheme of the redistribution is shown in three steps: $(a)$ the AUC for the Penrose tiling without phason flips; (b) part of the distribution representing points $O$ participating in the flip is cut and shifted (marked with red) if the flip occurs; $(c)$ the AUC after the phason flip.
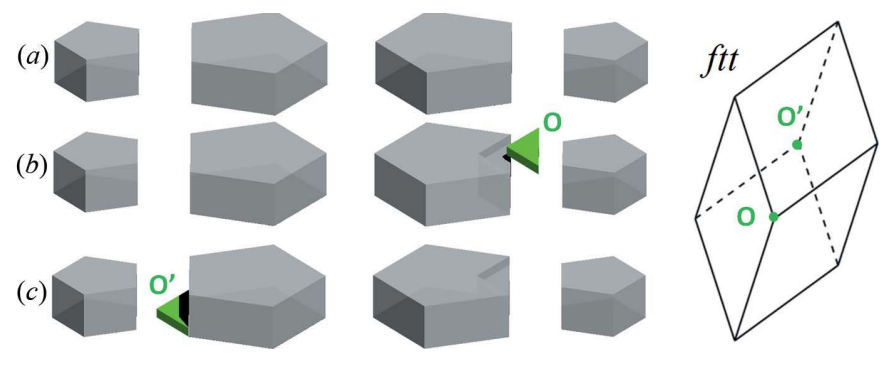

Figure 4

The same scheme as in Fig. 3 presented for the patch fft with the green prism being shifted to the new position after the phason flip.
(Wolny et al., 2016). Since the flip makes the $O$ positions unoccupied and the node of the Penrose tiling is shifted to the $O^{\prime}$ positions, which previously were empty, part of the distribution in the AUC is cut out from the region of the $O$ position and is shifted to the region corresponding to the $O^{\prime}$ node. The distribution is non-zero in a new region of the $O^{\prime}$ positions. The same reasoning can be applied to the other flip scenario: a pack of two thin rhombuses and one fat ( $f t$ - fat, thin, thin), as presented in Fig. 4. In this case, a different part of the AUC distribution is changed. The representation of the tiling with phason flips in the form of the distribution is much more complex in the case of an arbitrary decoration. It is then necessary to separate the distributions of the thick and thin rhombuses. The distribution of the reference vertex of the rhombus is a triangle in the distribution space (AUC or perpendicular space). Any deviation from the perfect Penrose tiling ordering will affect the shape of the triangle.

In the following, we provide a derivation of the analytical shape of the reference vertex distribution of the rhombus depending on which phason flip it is subjected to. The subdivision must be finer than for the Penrose tiling without flips. This time, the rhombus can appear locally in three different flippable patches. An explanation will be provided for the thick rhombus $L_{23}$, and is analogous for other types and orientations of rhombuses. A construction of the AUC subdivision is shown in Fig. 5 for $L_{23}$ and in Fig. 6 for $S_{24}$ (red indicates the $f f t$ flip scenario and green is the $f t t$ flip scenario). The first flippable pattern corresponds to the pack of rhombuses $L_{23}, L_{12}$ and $S_{13}$. It is labelled $12+23$ in Fig. 5. The reference vertex in this case is the initial point of vectors $\mathbf{d}_{1}, \mathbf{d}_{2}$, $\mathbf{d}_{3}$, spanning all rhombuses of the pack. The second flippable pattern is observed for $L_{23}, L_{34}$ and $S_{24}$ (the triangular region labelled $23+34$ in Fig. 5). The last pack of flippable rhombuses is $L_{23}, S_{25}$ and $S_{35}$. In this case, the label 23 is sufficient to determine the flippable pack and the orientation of the $L$ rhombus. In all cases, the reference vertex of the distribution is the reference vertex of the thick rhomb $L_{23}$. After the flip, part of the distribution from a region corresponding to a specific flip is shifted to the determined region of the other pentagon. The volume of the distribution which is shifted is proportional to the flip probability. This means that if the flips $L f t t 23$ with probability $\alpha=0.2$ are considered, $20 \%$ of the height from the corresponding region is cut out uniformly and shifted to the second pentagon, to the region of the distribution $L$ ftt 23 on the second pentagon. The same can be derived for the thick rhombus $L_{23}$ from the second pentagon. This time, part of the distribution is shifted to the first pentagon after the flip. The same is applicable for the thin rhombus. All possibilities for one selected orientation of each rhombus $\left(L_{23}\right.$ and $\left.S_{24}\right)$ are presented in Figs. 5 and 6 . It is important that the structure of the Penrose tiling with phason flips can have a distribution outside the pentagonal border of the atomic surface (or equally the AUC). This was true in the case of a nondecorated tiling (region $O^{\prime}$ in Figs. 3 and 4), but it is also the case for the distributions of individual rhombuses.

The statistical description of the structural disorder (the phasons) in the quasilattice is only an approximation. In this 



Figure 5

The subdivision of the AUC into regions representing local configurations of the $L_{23}$ rhombus in the Penrose tiling depending on the local environment. The regions of the $f f t(f t t)$ patch are shown in red (green). The distributions before and after the flip are shown in the top and bottom parts of the image, respectively. The left (right) panel shows the flip of the rhombus from the first (second) pentagon of the atomic surface. A new region in the distribution can appear outside the pentagon.

picture, the location of the flip in a real structure has no effect on the diffraction pattern. Only a parameter that can be associated with the phason-flip probability is important. This seems farfetched, but it is easy to understand when we take a closer look at the distribution of the nodes of the Penrose tiling. The distribution has the same shape with no relation to the place where the flip is introduced as long as the structure is infinite and the flip is really random; that is, we do not assume any local environment that the flip is capable of happening in. For the finite structure, a difference in the diffraction intensity will be observed that depends on the place of the flip, resulting in broadening of the correlation plot (Wolny et al. 2016). Another comment we must make is that we consider flips that can occur in the ordered Penrose tiling. If the site is already flipped, rearranged tiles do not participate in further flips. This is a kind of hidden correlation violating the rule of pure randomness; however, the derived approximation seems to be satisfied as the probability that there could be a consecutive flip from an already flipped patch is of the order of $\alpha^{2}$. For small structural disorder, the effect of this hidden correlation is negligible. Our final comment on the model is that it describes a static structural disorder, rather than a dynamical effect of phason jumps. We consider that the Penrose tiling as a quasilattice can be modified by local mismatches from the perfect order; therefore, we add the effect coherently to the structure factor, and such an average structure is the subject of the refinement.
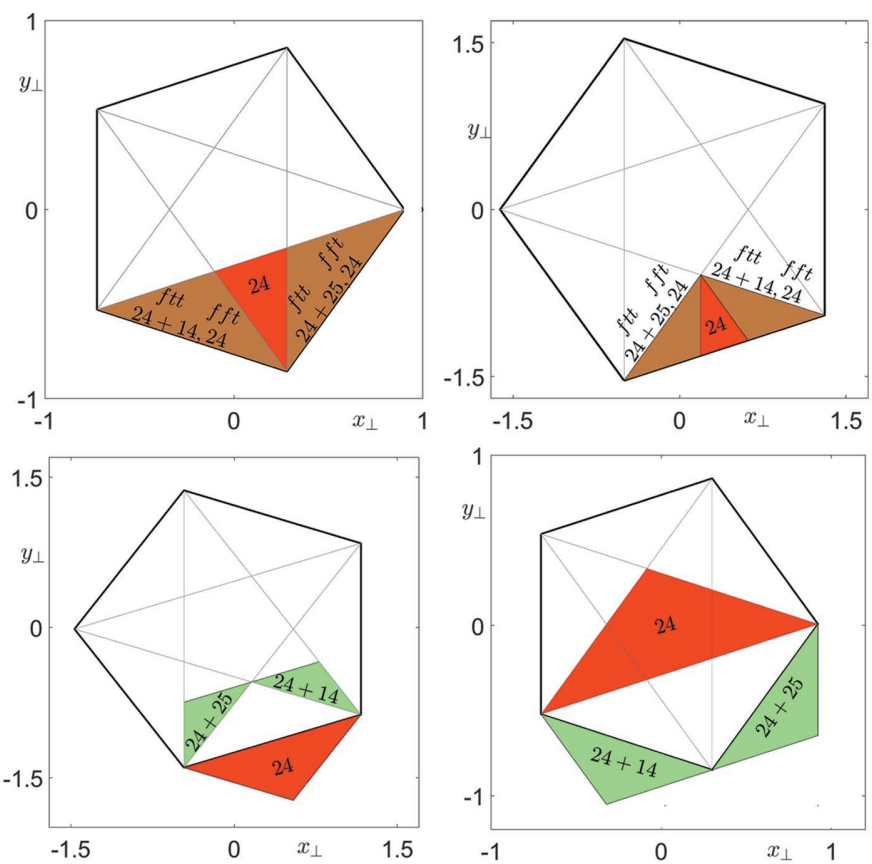

Figure 6

The subdivision of the AUC into regions representing local configurations of the $L_{23}$ rhombuses in the Penrose tiling depending on their local environment. The regions of the $f f t(f t t)$ patch are shown in red (green). Brown regions belong to both $f f t$ and $f t$ patches. In this case, the label ' 24 +14 ' denotes the ftt patch, whereas ' 24 ' denotes the fft patch. The distributions before and after the flip are shown in the top and bottom parts of the image, respectively. The left (right) panel shows the flip of the rhombus from the first (second) pentagon of the atomic surface. Again, a new region in the distribution can appear outside the pentagon.

\section{The structure of the AlCuRh decagonal quasicrystal}

A decagonal quasicrystal in the $\mathrm{AlCuRh}$ system was first investigated by Kuczera et al. (2012). The initial decoration of the Penrose tiling was found by phasing the diffraction pattern using the charge-flipping algorithm (Oszlányi \& Sütô, 2008) provided by SUPERFLIP (Palatinus, 2004; Palatinus \& Chapuis, 2007). The symmetry of the crystal was determined to be $P 10_{5} / m m c$, with a screw axis along the periodic direction of the crystal. The crystal is a periodic stacking of a pair of aperiodic planes (a double-period structure). The lattice constant in the periodic direction is $4.278 \AA$. A screw axis is realized by assigning two different decorations to each rhombus depending on its $z_{\perp}$ coordinate [corresponding to the position of the respective pentagonal distribution in the AUC (the atomic surface)] and whether it is a first or a second plane in the periodic direction. The decoration of the type-I rhombus [having the distribution of the reference vertex on the first pentagon of the AUC (the atomic surface)] in the second plane must be used to decorate the type-II rhombus (from the second pentagon) in the first plane with the decoration being inverted. The inversion of the decoration is particularly important in the case of the application of the phasonic correction. The decoration of the rhombus subjected to the flip must be inverted after each flip.

Kuczera et al. (2012) reported a refinement agreement factor of $R=7.87 \%$ for peaks larger than $1 \sigma$ (the structure- 
factor value is larger than its standard deviation). We performed a new refinement using equation (2) and all free parameters, including our new phasonic correction. The $R$ factor improved to $R=6.77 \%$ (Fig. 7) and the flip probability achieves $1.6 \%$ with a phasonic ADP, denoted as $b_{\mathrm{ph}}$, of $1.27 \AA^{2}$. The conclusion is that $1.6 \%$ of all flippable configurations are changed in the refined structure according to the phason-flip rules. The probability of a secondary flip (rearrangement of atoms previously affected by the phason flip) was estimated at a negligible level of $0.026 \%$. The overall result confirms that our approach (of uncorrelated single phason flips) is correct. The effect of lattice structural disorder, however small, must be understood as an inherent feature of the structure, contributing to a change in the intensities of the diffraction peaks. Nevertheless, the smearing

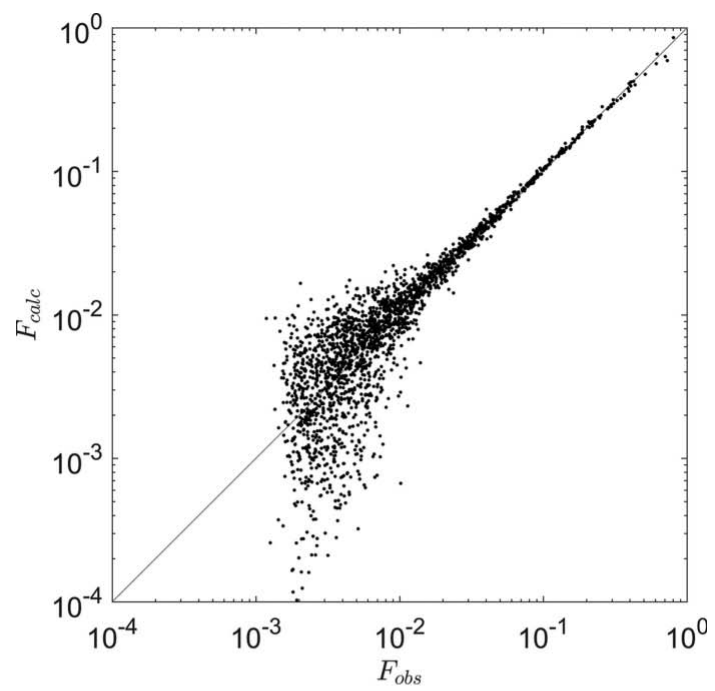

(a)

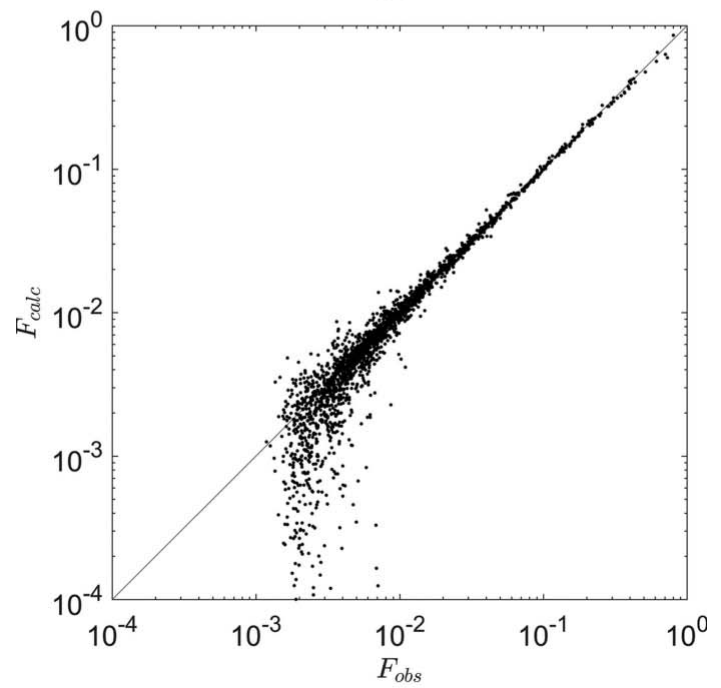

(b)

Figure 7

The $F_{\text {calc }}$ versus $F_{\text {obs }}$ plot for 2092 peaks with a lattice correction for phasons included solely $(a)$ and together with the standard phasonic Debye-Waller factor $(b)$. The agreement factors are $R=13.4 \%(a)$ and $R$ $=6.77 \%(b)$. If the phasonic Debye-Waller factor is not included in the refinement, the flip probability grows to an unphysical value of $\alpha=160 \%$. The Gaussian smearing of the atomic surface appears to be necessary. of the atomic surface is dominant. We calculated the standard deviation of the atomic displacement $u_{\mathrm{ph}}$ in the phason space as (with $b_{\mathrm{ph}}$ being the phasonic ADP)

$$
\left\langle u_{\mathrm{ph}}\right\rangle=\left(b_{\mathrm{ph}} / 8 \pi^{2}\right)^{1 / 2} \text {. }
$$

The value of $u_{\mathrm{ph}}$ can be compared with the diameter of the atomic surface $(\sim 1.5)$. The difference is significant - the phason space displacement is ten times smaller than the size of the atomic surface. To give a better perspective on the meaningfulness of the lattice phason with respect to the Gaussian smearing of the atomic surface associated with the phasonic Debye-Waller factor, we have plotted a convolution of the atomic surface with a Gaussian distribution. The plot presented in Fig. 8 was created for the atomic surface of the Fibonacci chain, which is simpler to represent. Even though the shape of the atomic surface for the decagonal quasicrystal is different, the simplistic example of the Fibonacci chain explains the relation between the lattice phason and the phasonic ADP well. The lattice phason flip ratio and the phasonic standard deviation were taken from the result of the $\mathrm{AlCuRh}$ refinement. The green dashed line shows the convolution of the Gaussian distribution with the atomic surface without the lattice phasons. The continuous line represents the same convolution but with $1.6 \%$ of the phason flips introduced to the atomic surface. We can see that although the difference in the smearing effect of the Gaussian with or without phasons is very small, it is noticeable. We reported previously that even a very small phason-flip probability could lead to a characteristic bias in the logarithmic plot of calculated versus measured intensities (Buganski et al., 2015). Even though the effect is significantly diminished by Gaussian smearing, a small difference still exists which can potentially affect the weak reflections. All the refined parameters can be accessed in the supporting information.

The correction for phasons was, in general, used together with the phasonic Debye-Waller factor. After the final refinement, we performed a test to check whether not

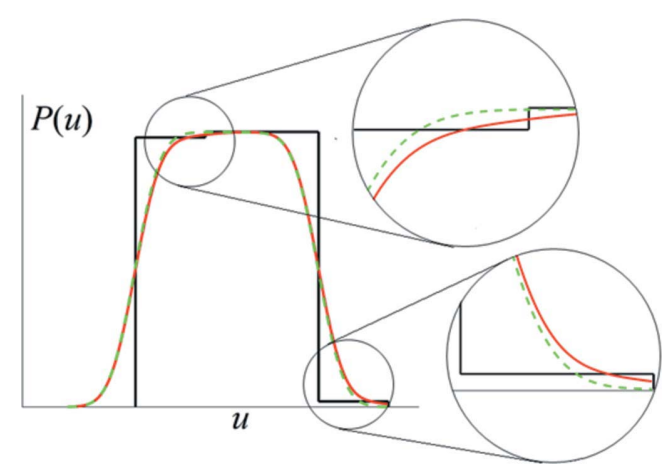

Figure 8

The smearing of the atomic surface in the Fibonacci chain. The distribution for the Fibonacci chain with $1.6 \%$ phason flips (black) is compared with the convolution with a Gaussian function $(\sigma=10 \%$, red) and the convolution of the same Gaussian function for the structure without flips (green dashed line). A small difference is seen, showing that within a given phasonic Gaussian function the effects of the phason lattice flips can be seen. 

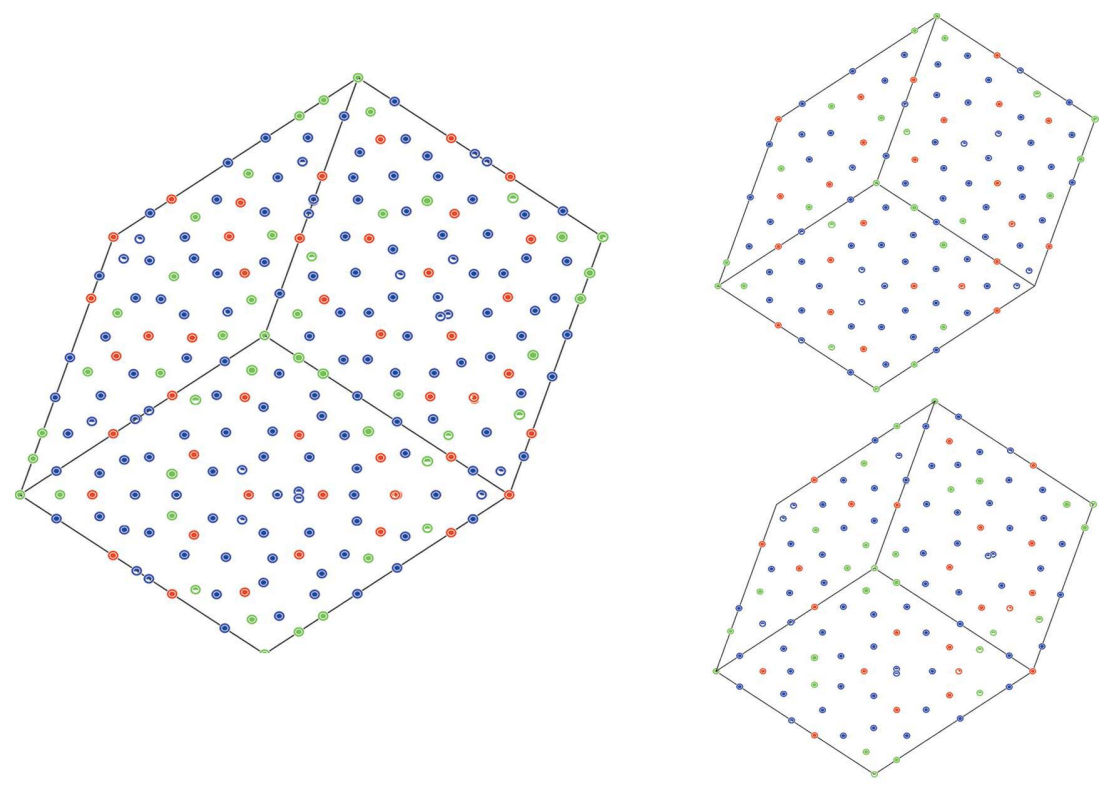

crystals. Random phason flips of individual atoms, possibly corrected by the Gaussian term, must be also included in the refinement. The resulting correlation plot is given in Fig. 7(a). A strong agreement is observed up to a range of $10^{-2}$ on a relative scale of diffraction amplitudes. This proves once again that small peaks are essential for a proper description of the quasicrystalline structure. A deeper meaning of the general Debye-Waller factor and justification of its applicability in the structure determination of quasicrystals await further studies.

In the next part, we discuss a problem of the matching rules for tiling under the phasonic disorder. The question is whether the matching rules are violated after the phason flips or not. If the matching rules are violated, each phason flip would lead to infinite energy cost, and the refined structure would be clearly unphysical.
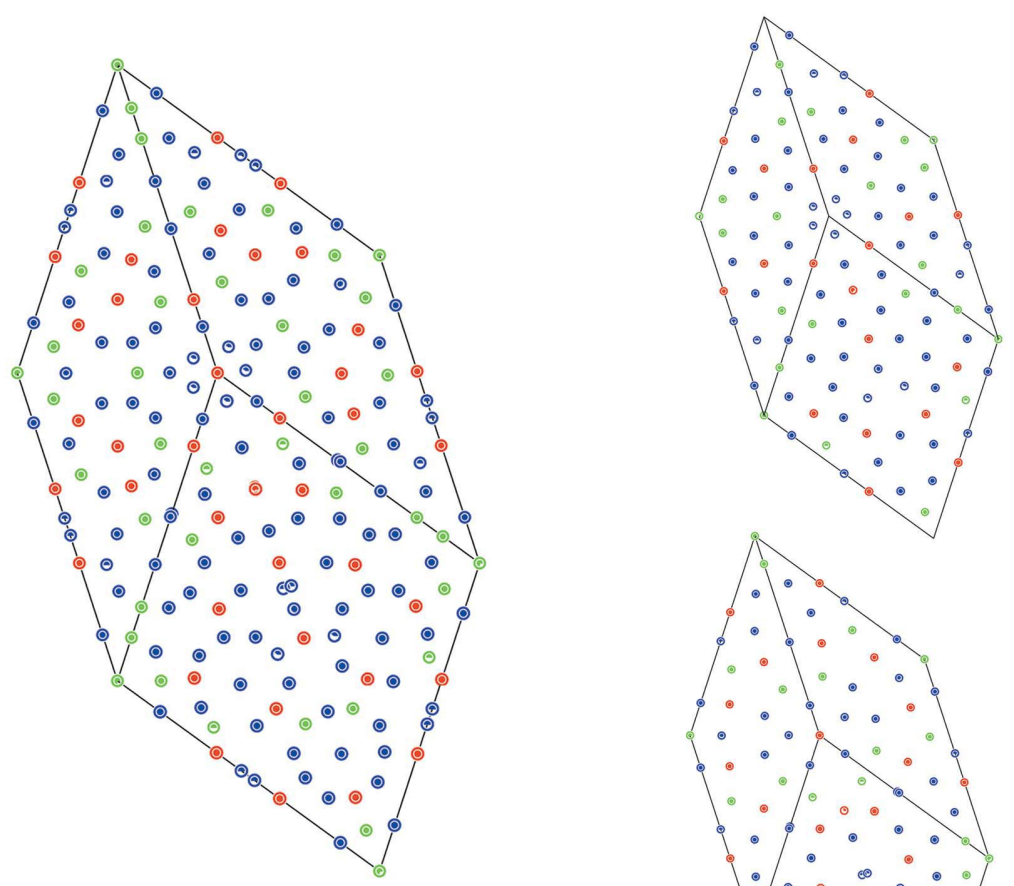
Fig. 9 shows the tiles assembled to a flippable patch. The condition of the preservation of the matching rules is to have the same decoration of outer edges of the flippable patch before and after the flip. If we take a look at the lefthand part of Fig. 9, we can see two fft and ftt patches assembled from decorated rhombuses. In this image, atoms from the second and first plane are projected together. It is easy to see that the decoration of edges is invariant under the inversion, and since the flipped pattern is an inversion of the original this means the matching rules are not violated. Let us inspect the flippable pattern at each of the atomic planes (Fig. 9, right). One can find an apparent contradiction in the decoration of the edges before and after a flip. The decoration of the edges does indeed change after inversion in an individual plane, but finds its equivalence in another plane. The decoration of the edges in a flipped version on the first plane is the same as the decoration formed on the second plane. The interchange of atoms must happen not only in plane but also in between planes. Keeping that in mind, it must be emphasized that our approach to phasons should not be understood as a modelling of dynamical effects. We interpret this phason-flip model as the realization of static disorder occurring in the structure, where the ordering of the

Figure 9

The atomic decoration of flippable patches in the $d$-AlCuRh quasicrystal. Red: Rh, green: $\mathrm{Cu}$, blue: Al. The two-layer projection (left) and decoration of each of the two planes (right) are shown. The length of the tile edge is $17.19 \AA$.

including the phasonic Debye-Waller factor is possible in our approach. When the value of the phasonic ADP $b_{\mathrm{ph}}$ was set to 0 , an unphysical value of the flip probability of $160 \%$ was obtained together with a significantly worse $R$ factor of $13.4 \%$. This might be an indication that the inclusion of the lattice part of the phason disorder solely in the refinement is insufficient in a discussion of the phason phenomena in quasi-
Penrose tiling is not strictly kept, but occasionally (in $1.6 \%$ of all cases) the tiles are flipped.

It must be emphasized that all the parameters, including ADPs and atomic positions, were released during the refinement. This resulted in a statistically significant improvement of the model compared with that previously reported by Kuczera et al. The atomic composition was refined to be 
$\mathrm{Al}_{61.1} \mathrm{Cu}_{17.6} \mathrm{Rh}_{21.3}$, with slightly higher $\mathrm{Rh}$ and smaller $\mathrm{Cu}$ content with respect to the experimental data (Kuczera et al., 2012). We assumed the same value of the phason-flip probability for all possible flips (flip $f f t$ and $f t$ ) in all orientations to avoid an increase in the number of parameters to be refined in the phason model. In general, different probabilities could be assigned to the different flip scenarios, even assuming orientational anisotropy. The refined atomic positions, ADPs and phasonic coefficients were responsible for most of the improvement. Nevertheless, the bias in the calculated peak intensities still prevails; we discuss this problem in the next section.

\section{Refinement with intensity redistribution}

The existence of the bias in the calculated intensities is most likely the effect of multiple scattering, which is dominant for the weak reflections. This hypothesis, which exists in the community, is the only alternative to the phasons hypothesis so far (Fan et al., 2011; Takakura \& Mizuno, 2015). The mechanism of multiple scattering can be described in the following way. An incident X-ray beam is diffracted in two or more directions which are not parallel to each other. As a consequence, the intensity of the diffracted beam is divided into primary and secondary beams. This leads to a weakening in the intensity of a (primary) beam detected for a reflection observed at a given diffraction geometry. The primary reflected beam acts as an incident beam for the secondary reflection. This happens only if all reciprocal-lattice points for which the scattering occurs lie on the Ewald sphere (Rossmanith, 2004). Since the reciprocal space of quasicrystals is dense, multiple scattering is very strong for these materials. The loss of the incident intensity results in the weakening of the primary peak and the enhancement of secondary peaks. In order to properly calculate the magnitude of the multiplescattering effect, the geometry of the experiment must be known and all reciprocal-space points that lie on the Ewald sphere during a measurement must be identified. A proper correction for multiple scattering in the case of periodic crystals was implemented by Rossmanith (1999) within the UMWEG99 software based on Renninger's result (Renninger, 1937). The data we have were collected in 2012 and we cannot repeat Rosmanith's procedure. Instead of using the kinematical approach, we decided to treat the problem phenomenologically, taking into account that the effect of multiple scattering changes the ratio of intensities between peaks. We assumed that during the experiment there exists a geometry that makes all the collected peaks interact with each other. We do not assume that all peaks always lie on the Ewald sphere; rather we claim that during the scanning of the reciprocal space any peak from the data finds itself on the Ewald sphere with every other peak at some point during the experiment. Furthermore, we assume that a certain fraction of the intensity is being transferred with a constant probability $\gamma$ for all peaks. The formula for the diffraction intensity influenced by multiple scattering is

$$
\begin{aligned}
\mathrm{d} I_{\text {calc }}^{\mathrm{I}}\left(\mathbf{k}^{\prime}\right) & =\gamma \sum_{\mathbf{k} \in \text { data } \neq \mathbf{k}^{\prime}}^{N} I_{\text {calc }}(\mathbf{k}), \\
\mathrm{d} I_{\text {calc }}^{\mathrm{II}}\left(\mathbf{k}^{\prime}\right) & =-\gamma(N-1) I_{\text {calc }}\left(\mathbf{k}^{\prime}\right), \\
I\left(\mathbf{k}^{\prime}\right)_{\text {calc }}^{\text {mult }} & =I_{\text {calc }}\left(\mathbf{k}^{\prime}\right)+\mathrm{d} I_{\text {calc }}^{\mathrm{I}}\left(\mathbf{k}^{\prime}\right)+\mathrm{d} I_{\text {calc }}^{\mathrm{II}}\left(\mathbf{k}^{\prime}\right),
\end{aligned}
$$

where $N$ is the number of peaks, $\mathrm{d} I_{\text {calc }}^{\mathrm{I}}\left(\mathbf{k}^{\prime}\right)$ is an increase in the intensity of the peak for the wavevector $\mathbf{k}^{\prime}$ caused by multiple scattering, $\mathrm{d} I_{\text {calc }}^{\mathrm{II}}\left(\mathbf{k}^{\prime}\right)$ is a depletion of the intensity and $I\left(\mathbf{k}^{\prime}\right)_{\text {calc }}^{\text {mult }}$ is the value of the calculated intensity for the peak associated with the scattering vector $\mathbf{k}^{\prime}$. Equation (4) must be used for every peak in the diffraction diagram. According to equation (4), part of the intensity belonging to each peak is redistributed to different peaks, keeping in mind that the total energy is preserved. This means that the same amount of intensity transferred to other peaks is also subtracted from the original peak. We limit the redistribution to the asymmetric part of the reciprocal space, as the multiplicity factor would only affect the $\gamma$ value.

Equation (4) is an empirical formula introduced as a simple tool for dealing with the intensity redistribution. The full analysis of the multiple scattering would require an integration of the peak intensity for every $\psi$ and $\omega$ measurement angle (Rossmanith, 2006). Additionally, the interference between the primary and secondary beam would need to be included [Klein \& Furtak (1988), which is a translation of Klein \& Furtak (1986)]. Since we do not know the geometry of the experiment, the angle between the primary and secondary beam is unknown, and the full analysis cannot be performed in our case.

The refinement procedure including multiple scattering was as follows: first we fitted the parameter $\gamma$ from equation (4), keeping other parameters the same except for the phason-flip probability and the phasonic Debye-Waller factor. The agreement factor was $R=6.10 \%$ and the bias in the calculated peaks disappeared. It is interesting that we can observe a substantial increase of the phasonic correction in the general Debye-Waller factor (the phasonic ADP increases from 1.265 to $1.350 \AA^{2}$ ) and a decrease of the phason-flip probability (from $1.6 \%$ to $1.34 \%$ ). This might be an effect of the approximation used for multiple scattering. At the end, we attempted to refine all parameters together, but the improvement of the $R$ factor was rather low (by around $0.1 \%$ to $R=6.00 \%$ ). The refinement result is shown in Fig. 10 . Although the characteristic bias is no longer present in the plot, the refinement result is not much better, except for the significant change in the disorder parameters. Because the final structural model has not changed, the improvement of the refinement result due to the correction for multiple scattering itself could appear to be artificial. However, we should keep in mind that according to Fig. 7, up to a certain level, even a strong change in the shape of the occupation domain leads to the correct refinement result. Only weak peaks give a clear distinction between a less or more probable structural model. Our corrective term allows us to get rid of the bias and does not affect the correctness of the result, and at the same 


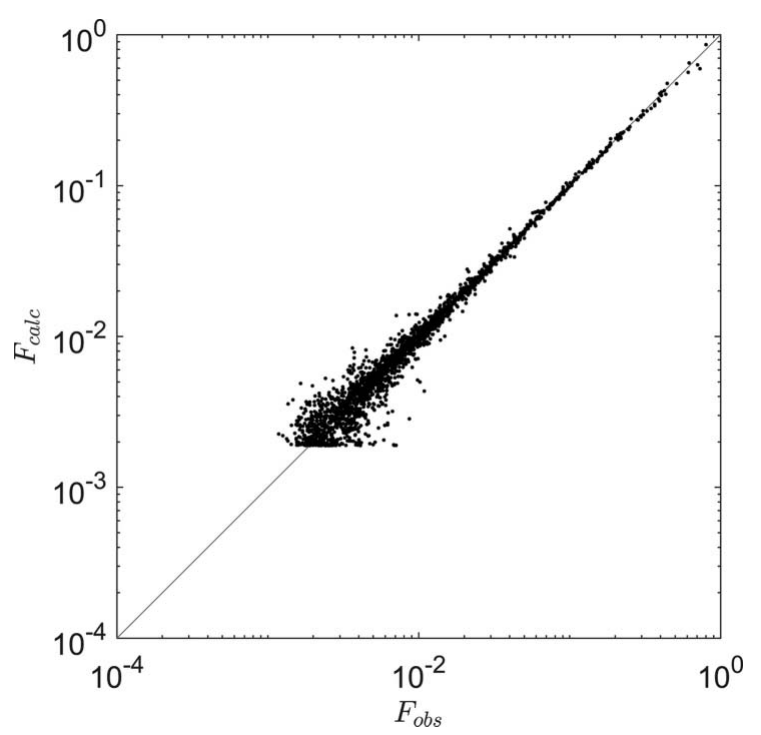

Figure 10

$F_{\text {calc }}$ versus $F_{\text {obs }}$ plot for 2092 peaks of the AlCuRh decagonal system after the application of the quasilattice phasons and multiple-scattering correction. All parameters were released during the refinement. The agreement factor was $R=6.00 \%$.

time supports the idea that such an empirical correction could also be successfully applied in other cases.

\section{Summary}

We have investigated the effect of phason flips, as discussed for the atomic structure of a decagonal quasicrystal built upon the Penrose tiling. We also introduced a peak-intensity redistribution correction that solves the problem of multiple scattering in the structure refinement. The structure factor with phason flips was derived assuming a random phason flip in a quasilattice of the Penrose tiling. Only phason flips of the first order were considered because of the assumption of a weakly disordered structure. The regions of the distribution of rhombuses in the perpendicular (or the AUC) space were found and analytically defined, which allowed calculation of the structure factor for an arbitrary tile decoration. The new corrective term was tested against the real structure of the decagonal $\mathrm{AlCuRh}$ quasicrystal, for which the best quality data were accessible. The correction is used, together with the generalized Debye-Waller factor, to include a random rearrangement of atoms. We managed to significantly improve the final result of the refinement and achieved a satisfactory agreement with the experimental data with an $R$ factor of $6.77 \%$. The new parameter providing information about the phason-flip probability was refined to be $1.6 \%$. At this stage, we can positively conclude that the bias is not caused solely by the phason disorder. To test the alternative hypothesis on possible reasons for the characteristic bias in the refinement results of quasicrystals, namely multiple scattering, we introduced another corrective term. It was a sample- and experiment-geometry-independent correction which assumed a redistribution of peak intensities with a constant probability coefficient. The application of the correction for multiple scattering to the $\mathrm{AlCuRh}$ system resulted in a significant improvement of the agreement factor. Also, the characteristic bias in the logarithmic plot of the calculated and the measured intensities was explained. The final refinement, with all the parameters being released, resulted in a minor change in the values of the parameters except for the value of the phasonic ADP and the phason-flip probability. The data quality is apparently strongly correlated with the phason disorder. A fully qualitative analysis of the phason disorder is beyond our capabilities because of the insufficient quality of the data and unknown geometry of the diffraction experiment. However, we demonstrated that significant improvement can be achieved by applying the empirical correction formula for multiple scattering and the new approach to phason flips.

\section{Funding information}

The following funding is acknowledged: Narodowym Centrum Nauki (grant No. 2016/21/N/ST3/00287 to Ireneusz Buganski); Ministry of Science and Higher Education (Poland) (subsidy No. 11.11.220.01/5 to Faculty of Physics and Applied Computer Science).

\section{References}

Abe, E. \& Tsai, A. P. (2001). JEOL News, 36E, 18-21.

Bancel, P. A. (1989). Phys. Rev. Lett. 63, 2741-2744.

Bruijn, N. G. de (1981). I. Indagat. Math. 84, 39-52.

Buganski, I., Chodyn, M., Strzalka, R. \& Wolny, J. (2017). J. Alloys Compd. 710, 92-101.

Buganski, I., Strzalka, R. \& Wolny, J. (2015). Phys. Status Solidi B, 253, 450-457.

Cascarano, G., Giacovazzo, C., Camalli, M., Spagna, R. \& Watkin, D. J. (1991). Acta Cryst. A47, 373-381.

Dąbrowska, A., Kozakowski, B. \& Wolny, J. (2005). Acta Cryst. A61, 350-357.

Duda, M., Kozakowski, B. \& Wolny, J. (2007). J. Non-Cryst. Solids, 353, 2500-2505.

Duneau, M. \& Katz, A. (1985). Phys. Rev. Lett. 54, 2688-2691.

Fan, C. Z., Weber, T., Deloudi, S. \& Steurer, W. (2011). Philos. Mag. 91, 2528-2535.

Henley, C. L. (1986). Phys. Rev. B, 34, 797-816.

Henley, C. L., Elser, V. \& Mihalkovic, M. (2000). Z. Kristallogr. 215, 553-568.

Janssen, T. \& Radulescu, O. (2003). Ferroelectrics, 395, 179-184.

Kiselev, A., Engel, M. \& Trebin, H.-R. (2012). Phys. Rev. Lett. 109, 225502.

Klein, M. V. \& Furtak, Th. E. (1986). Optics. New York: John Wiley.

Klein, M. V. \& Furtak, Th. E. (1988). Optik. Berlin, Heidelberg: Springer.

Kozakowski, B. \& Wolny, J. (2010). Acta Cryst. A66, 489-498.

Kuczera, P., Wolny, J. \& Steurer, W. (2012). Acta Cryst. B68, 578-589.

Kuczera, P., Wolny, J. \& Steurer, W. (2014). Acta Cryst. B70, 306-314.

Lubensky, T. C., Socolar, E. S., Steinhardt, P. J., Bancel, P. A. \& Heiney, P. A. (1986). Phys. Rev. Lett. 57, 1440-1443.

Ors, T., Takakura, H., Abe, E. \& Steurer, W. (2014). Acta Cryst. B70, 315-330.

Oszlányi, G. \& Sütő, A. (2008). Acta Cryst. A64, 123-134.

Palatinus, L. (2004). Acta Cryst. A60, 604-610.

Palatinus, L. \& Chapuis, G. (2007). J. Appl. Cryst. 40, 786-790.

Panjikar, S., Parthasarathy, V., Lamzin, V. S., Weiss, M. S. \& Tucker, P. A. (2009). Acta Cryst. D65, 1089-1097.

Pavlovitch, A. \& Kleman, M. (1987). J. Phys. A Math. Gen. 20, 687702. 
Penrose, R. (1974). Bull. Inst. Math. Appl. 10, 266-271.

Renninger, M. (1937). Z. Phys. 106, 141-176.

Rossmanith, E. (1999). J. Appl. Cryst. 32, 355-361.

Rossmanith, E. (2004). J. Appl. Cryst. 37, 493-497.

Rossmanith, E. (2006). Acta Cryst. A62, 174-177.

Shechtman, D. S., Blech, I., Gratias, D. \& Cahn, J. W. (1984). Phys. Rev. Lett. 53, 1951-1953.

Skubák, P. \& Pannu, N. S. (2013). Nat. Commun. 4, 2777.

Steurer, W. (2018). Acta Cryst. A74, 1-11.

Steurer, W. \& Deloudi, S. (2009). Crystallography of Quasicrystals. Concepts, Methods and Structures. Berlin: Springer.
Takakura, H., Gómez, C. P., Yamamoto, A., de Boissieu, M. \& Tsai, A. P. (2007). Nat. Mater. 6, 58-63.

Takakura, H. \& Mizuno, R. (2015). Mater. Struct. 22, 281.

Taniguchi, S. \& Abe, E. (2008). Philos. Mag. 88, 1949-1958.

Wolny, J. (1998). Philos. Mag. A, 77, 395-412.

Wolny, J., Buganski, I., Kuczera, P. \& Strzalka, R. (2016). J. Appl. Cryst. 49, 2106-2115.

Wolny, J., Buganski, I. \& Strzalka, R. (2017). Crystallogr. Rev. 24, 143.

Wolny, J., Kozakowski, B. \& Repetowicz, P. (2002). J. Alloys Compd. 342, 198-202. 